\title{
Osteoarthritis and psoriatic arthritis: Findings in three-dimensional biophotonics imaging
}

\author{
Zhen Yuan $^{\mathrm{a},{ }^{*}}$, Fong H. Ieong ${ }^{\mathrm{a}}$, Huabei Jiang ${ }^{\mathrm{b}}$ and Eric S. Sobel ${ }^{\mathrm{c}}$ \\ ${ }^{a}$ Bioimaging Core, Faculty of Health Sciences, University of Macau, Macau SAR, China \\ ${ }^{b}$ Department of Biomedical Engineering, University of Florida, Gainesville, FL 32611, USA \\ ${ }^{c}$ Division of Rheumatology, College of Medicine, University of Florida, Gainesville, FL 32611, USA
}

\begin{abstract}
The goal of this study is to capture the quantitative optical features of degenerative finger joints based on $\mathrm{x}$-ray aided three-dimensional (3D) diffuse optical tomography (DOT). It is anticipated that the fused imaging technique can be applied to identifying the significant differences between osteoarthritis (OA) and psoriatic arthritis (PA). For a case study, total 6 subjects were selected to study the distal interphalangeal (DIP) finger joints. 2 OA patients, 2 PA patients and 2 healthy subjects were examined clinically first. Their DIP finger joints were then scanned by the multimodality imaging method. Our findings suggested that the developed multimodality imaging approach may aid to contradistinguish OA patients from PA patients with the healthy control, which is essential for a better diagnosis and treatment of inflammatory arthritis in humans.
\end{abstract}

Key words: diffuse optical tomography, osteoarthritis, psoriatic arthritis, x-ray

\section{Introduction}

Osteoarthritis (OA) affects nearly 60 million Americans [1,2] while the population of psoriasis is about 7.5 million in USA[3,4]. There have been estimated that $10 \%$ to $30 \%$ of patients with psoriasis will potentially progress with PA. PA generally first attacks the DIP finger joints [3, 6-8]. Likewise, there were also OA patients who might develop the severe disease in the DIP finger joints [8].

To diagnose bone and cartilage abnormalities as well as related soft tissues change influenced by OA and PA, vary imaging modalities have been established. They included ultrasound, x-ray, MRI, and CT [7,9-25]. Among the existing imaging methods, high-resolution x-ray is considered for diagnosis of joint-related diseases in most cases. However, x-ray is incapable of discriminating soft tissues very well due to its low contrast [26]. In contrast, DOT as an emerging non-ionizing technology has received much attention because the imaging contrast offered by DOT is high,

\footnotetext{
*Corresponding author: Zhen Yuan, Director of Bioimaging Core, Faculty of Health Sciences, University of Macau, Macau SAR, China. Tel.: 853-88224989; Fax: 853-8822-2314; E-mail: zhenyuan@umac.mo.
} 
suggesting a possible use in distinguishing between OA and PA. In a pilot study, it has showed that DOT is able to to detect early-stage OA in finger joints [27-32].

In the present study, we suggested that x-ray aided DOT imaging was capable of distinguishing significantly among OA and PA finger joints as well as healthy ones. We demonstrated the detailed radiographic presentations and functional optical properties of OA and PA patients. In our acknowledgement, this is the first investigation currently implemented to contrast the 3D optical findings between $\mathrm{OA}$ and $\mathrm{PA}$ finger joints.

\section{Participants and methodology}

\subsection{Participants and clinical examination}

In this clinical study, participants were enrolled between 2004 and 2009. The group of subjects consisted of volunteering patients with OA or PA and healthy individuals. The subjects needed sign the informed consent forms prior to taking part in this investigation, which was reviewed by the Research Committee in UF.

Each subject was examined and diagnosed to be with OA or PA by the same physician in UF. 2 PA patients and $2 \mathrm{OA}$ patients were classified respectfully. Exclusion criteria include the PA patients with clinical evidence of OA, and the patients whose clinical diagnostic of the diseased DIP figure joint was uncertainty. OA patients were selected by matching the OA clinical features and no additional arthropathies.

\subsection{Multimodal imaging systems}

The x-ray/ DOT system [30] fused an adjusted system of mini C-arm x-ray with a 64 by 64-channel DOT system (Figure 1(a)). The tomosynthetic image was generated via the mini C-arm x-ray system (MiniView 6800, GE-OEC, UT) [31].

The DOT system abided by a fiber optics/tissue interface, light detection modules, a hybrid light delivery subsystem, a data acquisition module, and laser modules $[29,30]$. The wavelength used in this study was $853 \mathrm{~nm}$.

The cylindrical fiber optics/tissue interface has 64 sources and 64 detector fiber bundles positioned in 4 layers. The space between a finger of the subject and the wall of the Plexiglas container was brimmed with tissue-like phantom materials. Coupling media consisted of agar powder, Indian Ink, distilled water, and Intralipid, which provides an absorption coefficient of $0.014 \mathrm{~mm}^{-1}$ and a reduced scattering coefficient of $1.0 \mathrm{~mm}^{-1}$ [29-31].

Along the cylindrical fiber optics/tissue interface, the subsystem comprising a motorized rotator and a 1x8 optical switch was used to transport laser light to the excitation points due to its efficient and low-cost hybrid nature (Figure 1(a)). The silicon-photodiodes coupled with programmable circuit boards, integrating the 64 low noises, were used for acquiring parallel signals. It took 5 minutes to finish the data acquisition based on this hybrid imaging system. 

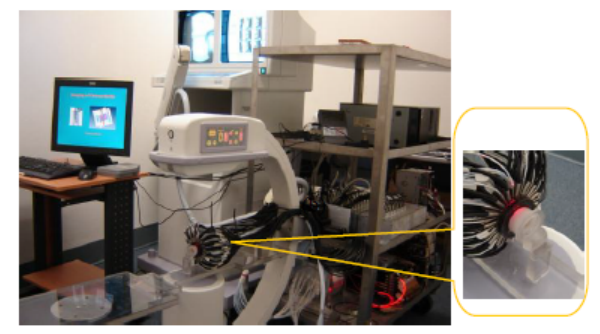

(a)

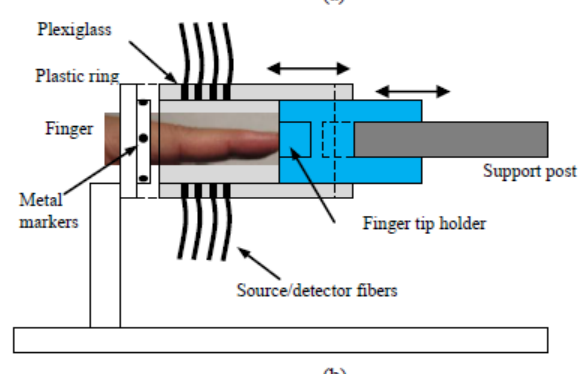

(b)

Fig. 1. (a) The fused $\mathrm{x}$-ray/DOT systems; (b) The fiber optics/x-ray interface.

In the hybrid imaging of soft tissues in the joint followed by the DOT data acquisition, the $\mathrm{x}$-ray imaging was performed instantaneously. A co-axial post was used to support the optical interface in order to eliminate the artifacts in the x-ray projections that might be possibly caused by the optical interface. Such way allowed the interface to be translated along the post as shown in Figures 1(a) and 1(b).

During an examination, the subject first placed his/her finger into the Plexiglas container through a plastic ring when the distal end of the finger was resting against a fingertip holder that was installed at the terminal of the co-axial post. The optical interface was then slid forth, contacting with the plastic ring structure that was used to fasten the position of the optical interface. It was then slid back for $\mathrm{x}$ ray exposure right after the DOT imaging while the position of the subject's finger stayed unchanged.

\subsection{Image analysis methods}

After data acquisition, the x-ray aided optical image was reconstructed based on a finite elementbased 3D reconstruction algorithm [30]. The 3D joint tomosynthesis enabled us to segment the imaging domain into 3 tissue types: (1) bone, (2) approximated soft tissues near the joint cavity and, (3) the phantom media. The known anatomy from 3D tomosynthesis images made it possible to localize the finite element nodes within each tissue. The entire image reconstruction cost about 4 hours with a $2 \mathrm{GHz}$ Pentium 4 PC. The joint space size was an important feature to quantify the seriousness of OA and PA. As such, a full width at half maximum (FWHM) method was adopted, in which representative dorsal (6 X-Z slices) and coronal slices (6 Y-Z slices) were segmented from the 3D images for further analysis to determine the joint space's thickness [30]. For each subject, the joint size determined by the optical images was provided, and the optical properties were also given for the 3 tissues. 


\section{Results}

To highlight the main optical reconstruction results, the analysis from 6 subjects (two OA/two PA patients, and two controllers) was focused.

\subsection{OA patients}

The first OA subject, female and aged 45, was diagnosed with OA in around 2010. The second female subject, aged 51 was diagnosed with OA in around 2004. Figure 2(a) shows the scattering slices while Figure 2(b) displays the absorption slices of the recovered 3D image for the second OA patient.

\subsection{PA patients}

For the first PA patient (48 year old), her DIP joint developed PA in around 2008. The second PA subject was a 56-year-old female diagnosed with PA in around 2004. Figure 3(a) presents the recovered optical scattering slices whereas Figure 3(b) plots the absorption ones of the first PA subject.

(a)
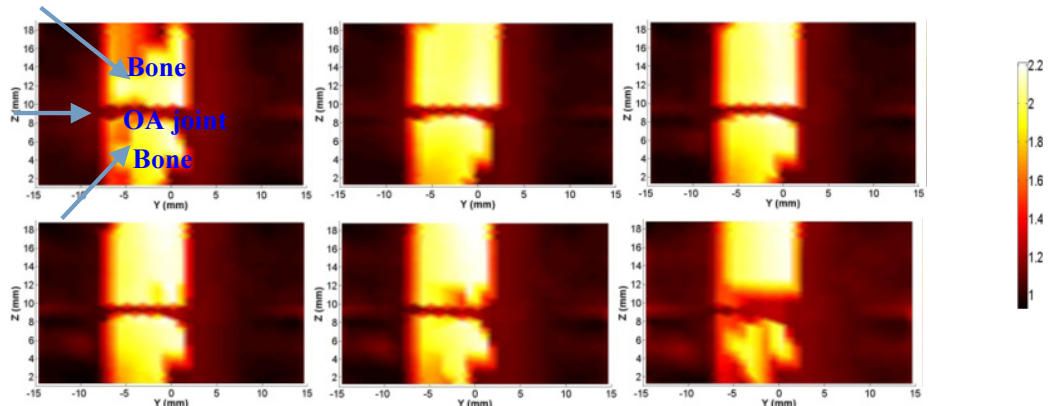

(b)
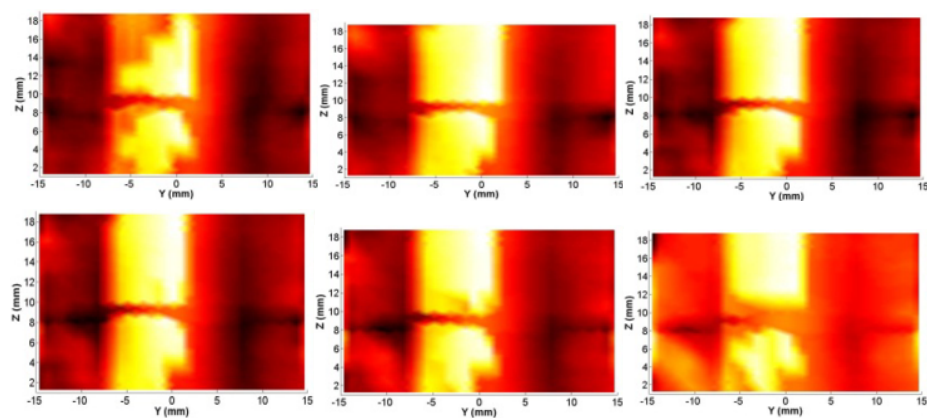

Fig. 2. Recovered images for OA patient 2: Scattering (a) and absorption slices (b). 

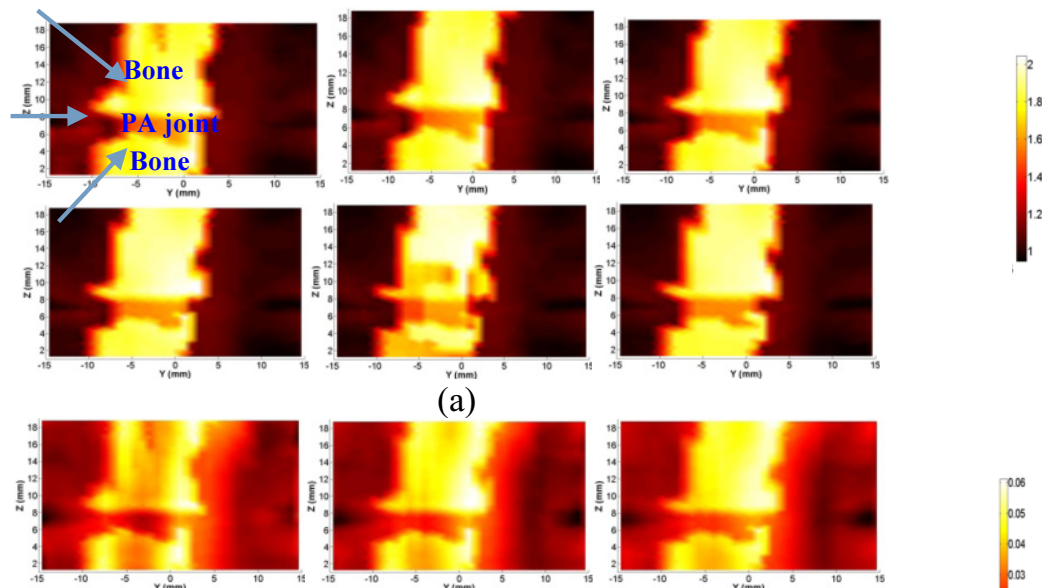

(a)
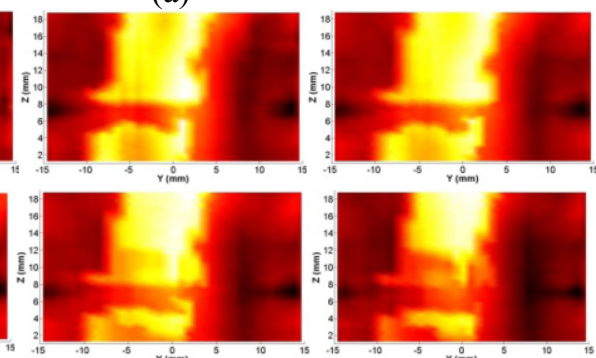

(b)

Fig. 3. Recovered images for PA patient 1: Scattering (a) and absorption slices (b).
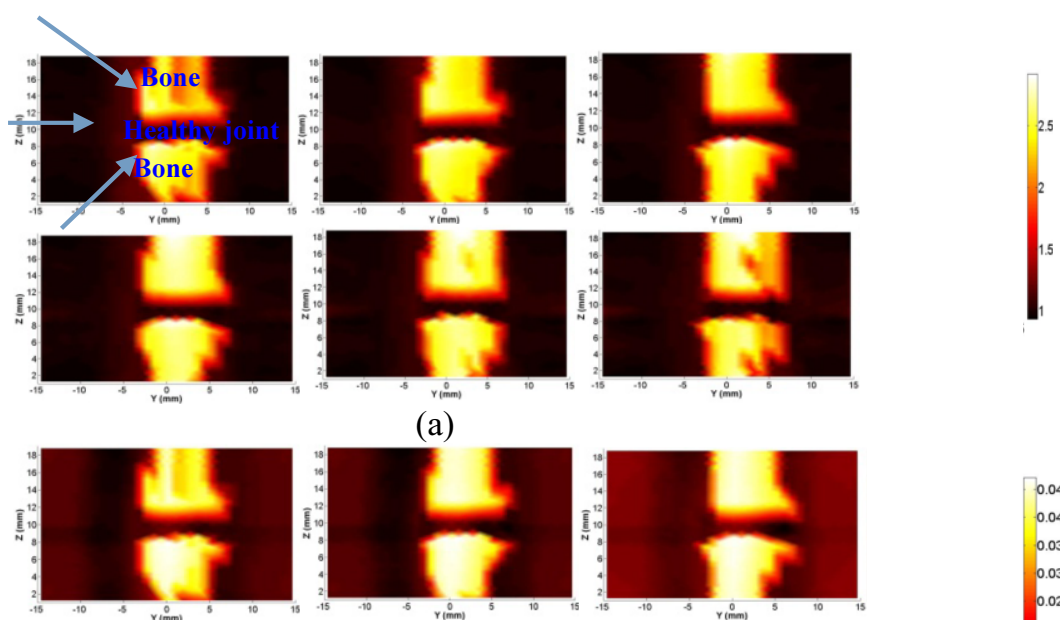

(a)
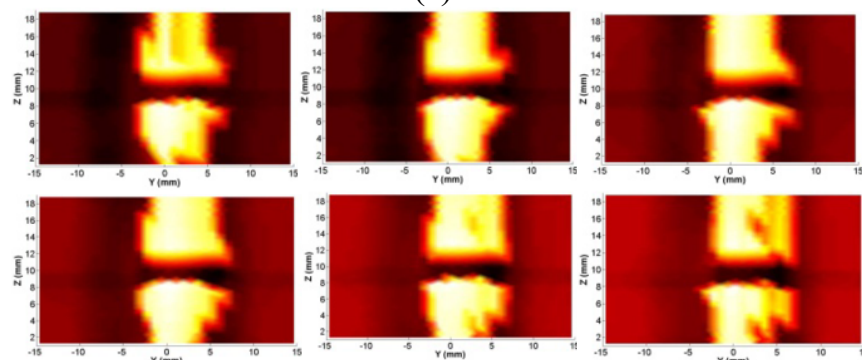

\begin{tabular}{|l|l|}
0.045 \\
0.04 \\
0.035 \\
0.03 \\
0.025 \\
0.02 \\
0.015 \\
0.01
\end{tabular}

$\int_{0}^{001}$

$$
\text { (1) }
$$




\subsection{Healthy volunteers}

The first healthy subject was a 41-year-old male while the second male controller was 43-year-old. Figures 4(a) and 4(b) show the reconstructed optical slices for the first healthy subject.

\subsection{Analysis of the recovered clinical results}

Based on the quantitative optical images in Figures 2-4, we found that there were significant differences between degenerative and healthy finger joints. Both the recovered optical absorption and scattering coefficients were able to distinguish well among OA, PA and normal DIP finger joints. In particular, a significant decrease of the optical properties of the soft tissues within healthy joints was observed while comparing with that of the bones. Nevertheless, we saw only a mild decrease in terms of the OA joint tissues. Interestingly the decrease of optical properties became even smaller from the PA joints than those from the OA ones. Our observations were also consistently with the quantitative evidences in Figure 5.

Interestingly, the difference in the reconstructed optical properties of the joint tissues among our subjects seemed more striking from the combined image feature in Figure 5. Its value was the product of ratios of optical absorption and scattering coefficients. We observed the combined image features for the disease joints were significantly greater than those for the normal joints. Moreover, the differences in the combined image features between the OA and PA joints estimated from the x-ray guided DOT reconstruction were also significant.

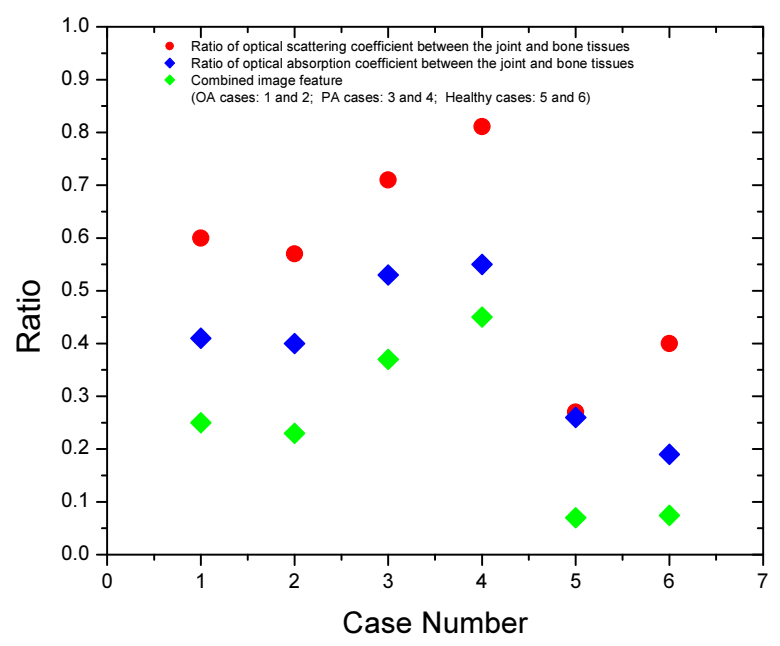

Fig. 5. The optical findings show the significant differences between PA, OA and healthy joints in terms of ratios of absorption (blue), scattering (red), and combined features (green). OA: case 1 and 2; PA: case 3 and 4; health subjects: case 3 and 4 . 
It should be pointed out that it was very challenging to distinguish firmly among the disease and control subjects in terms of the structural information in Figures 2-4. For instance, it seemed the narrowed joint space was a distinctive sign of OA while the structural changes in PA were evidenced by the joint, pencil-in-cup changes, and bone deformation.

\section{Discussion}

The examination of OA or PA depends on clinical findings and laboratory tests primarily, but sometimes it is difficult to distinguish OA joints from the PA ones. A radiologic comparison has been conducted to investigate the differences between erosive OA and PA [21]. Most clinicians usually classify patients for clinical management purposes according to number of joints involved and the presence or absence of enthesitis or spinal disease. In addition, the conventional radiography may show normality in OA or PA patients.

Besides one interesting MRI investigation [19], no study has been performed and compared the typical findings between patients with PA or OA and healthy finger joints. Case studies with 6 subjected including 2 OA subjects, 2 PA subjects and 2 controllers were implemented for this investigation. All the images were recovered using the existing multimodality imaging systems. Significant differences between degenerative (OA or PA) and healthy joints were revealed based on the observations of the ratio of optical parameters of joint tissues to those of the bones.

It is well known that the tissues involved most in OA are the joint soft tissues and subchondral bones. The vascular supply change and joint space narrowing will result in the increased optical properties of joint soft tissues. Importantly, the possible inflammatory change in OA will also increase the optical properties of the soft tissues surrounding the finger joints, including synovial fluid though the inflammatory is not so significant.

However, compared with OA joints, prominent inflammatory changes are observed for PA ones [19]. The significant inflammatory may result in an enhanced vascular supply feeding the PA joints. In addition, the significant inflamed joints in PA will result in the similarity in optical properties between the synovial fluid and neighboring tissues. These findings may be the main reasons why we observe an even smaller drop of optical properties for the soft tissues from PA joints. Interestingly, if a joint is a healthy one, a strong decrease of optical properties will be identified in the joint space area.

It is well-known significant difference of the mean optical properties is a key condition for the differentiation the diseased finger joints from healthy ones. Our data in terms of the optical properties of the DIP finger joints revealed the significant differences between (OA /PA) patients, and healthy subjects. These findings suggested the $\mathrm{x}$-ray guided DOT imaging is a convincing diagnostic mean for early detecting disease joints. A further investigation is required for both PA and OA based on large number of subjects.

\section{Acknowledgment}

This clinical study was supported partially by the National Institutes of Health (R01 AR048122) in US, SRG Grant (SRG2013-00035-FHS) and partially by the University of Macau (MYRG201400093-FHS Grant). 


\section{References}

[1] P. Sarzi-Puttini et al., Osteoarthritis: An overview of the disease and its treatment strategies, Semin, Arthritis Rheum 35 (2005), 1-10.

[2] S.B. Abramson, M. Attur and Y. Yazici, Prospect for disease modification in osteoarthritis, Nature Clinical Practice Rheumatology 2 (2006), 304-312.

[3] About psoriatic arthritis, available at http://www.psoriasis.org, July 18, 2014.

[4] Psoriatic arthritis, available at http://www.nlm.nih.gov/medlineplus/ency/article/000413.htm, July 18, 2014.

[5] D. Gladman, Current concepts in psoriatic arthritis, Current Opinion in Rheumatology 14 (2002), 361-366.

[6] H. Schoellnast et al., Psoriatic arthritis and rheumatoid arthritis: Findings in contrast-enhanced MRI, American Journal of Roentgenology 187 (2006), 351-357.

[7] P.A. Ory, D.D. Gladman and P.J. Mease, Psoriatic arthritis and imaging, Ann Rheum Dis. 64 (2005), ii55-ii57.

[8] D.J. Hart and T.D. Spector, Definition and epidemiology of osteoarthritis of the hand: a review, Osteoarthritis \& Cartilage 8 (2000), S2-S7.

[9] G. Karsenty, An aggrecanase and osteoarthritis, N. Engl. J. Med. 353 (2005), 522-523.

[10] S.S. Glasson et al., Deletion of active ADAMTS5 prevents cartilage degradation in a murine model of osteoarthritis, Nature 434 (2005), 644-648.

[11] C. Buckland-Wright, Radiographic assessment of osteoarthritis: comparison between existing methodologies, Osteoarthritis Cartilage 7 (1999), 430-433.

[12] L.C. Coates et al., Clues to the pathogenesis of psoriasis and psoriatic arthritis from Imaging: A literature review, The Journal of Rheumatology 35 (2008), 1438-1442.

[13] D. Mcgonagle, Imaging of the joints and enthesis: Insights into pathogenesis of psoriatic arthritis, Ann. Rheum. Dis. 64 (2005), ii58-ii60.

[14] P.J. Mease et al., Psoriatic arthritis assessment tools in clinical trials, Ann. Rheum. Dis. 64 (2005), ii49-ii54.

[15] W. Lai et al., Early diagnosis of osteoarthritis using cathepsin B sensitive near-infrared fluorescent probes, Osteoarthritis and Cartilage 12 (2004), 239-244.

[16] M. Backhaus et al., Arthritis of the finger joints, Arthritis \& Rheumatism 42 (1999), 1232-1245.

[17] I. Olivieri et al., The challenge of early diagnosis of psoriatic arthritis, The Journal of Rheumatology 35 (2008), 1-5.

[18] W.J. Taylor et al., A comparison of the performance characteristics of classification criteria for the diagnosis of psoriatic arthritis, Seminars in Arthritis and Rheumatism 34 (2004), 575-584.

[19] A.L. Tan et al., A high-resolution magnetic resonance imaging study of distal interphalangeal joint arthropathy in psoriatic arthritis and osteoarthritis, Arthritis \& Eheumatism 54 (2005), 1328-1333.

[20] M. Ostergaard et al., Magnetic resonance imaging-determined synovial membrane and joint effusion volumes in rheumatoid arthritis and osteoarthritis, Arthritis \& Eheumatism 40 (1997), 1856-1867.

[21] W. Martel et al., Erosive osteoarthritis and psoriatic arthritis: A radiologic comparison in the hand, wrist, and foot, American Journal of Roentgenology 134 (1980), 125-135.

[22] R.J. Wakefield, P.G. Conaghan, S. Jarrett and P. Emery, Noninvasive techniques for assessing skeletal changes in inflammatory arthritis: imaging techniques, Current Opinion in Rheumatology 16 (2004), 435-442.

[23] M.P. Spriet et al., Validation of a 40MHz B-scan ultrasound biomicroscope for the evaluation of osteoarthritis lesions in an animal model, Osteoarthritis Cartilage 13 (2005), 171-179.

[24] F. McQueen, M. Lassere and M. Ostergaard, Magnetic resonance imaging in psoriatic arthritis: A review of the literature, Arthritis Research \& Therapy 8 (2006), 207-214.

[25] M. Yoon, B. Choe and H. Chang, SU-FF-I-67: Magnetization transfer after bee venom acupuncture treatment to osteoarthritis, Med. Phys. 33 (2006), 2012-2016.

[26] J.T. Sharp, Assessment of radiographic abnormalities in rheumatoid arthritis: what have we accomplished and where should we go from here, J. Rheumatol. 22 (1995), 1787-1791.

[27] B.J. Tromberg, B.W. Pogue, K.D. Paulsen, A.G. Yodh and D.A. Boas, Assessing the future of diffuse optical imaging technologies for breast cancer management, Med. Phys. 35 (2008), 2443-51.

[28] Z. Yuan, Spatiotemporal and time-frequency analysis of functional near infrared spectroscopy brain signals using independent component analysis, J. Biomed. Opt. 18 (2013), 106011-1-106011-10.

[29] Z. Yuan, Q. Zhang, E. Sobel and H.B. Jiang, 3D diffuse optical tomography imaging of osteoarthritis: Initial results in finger joints," J. Biomed. Opt. 12 (2007), 034001-1-034001-11.

[30] Z. Yuan, Q. Zhang, E. Sobel and H.B. Jiang, Tomographic x-ray-guided three-dimensional diffuse optical tomography of osteoarthritis in the finger joints, J. Biomed. Opt. 13 (2008), 044006-1-044006-10. 
[31] Z. Yuan, Q. Zhang, E. Sobel and H. Jiang, High resolution x-ray guided three dimensional diffuse optical tomography of joint tissues in hand osteoarthritis: Morphological and functional assessments, Med. Phys. 37 (2010), 4343-4354.

[32] Z. Yuan, X. Li and L. Xi, Listening to light scattering in turbid media: quantitative optical scattering imaging using photoacoustic measurements with one-wavelength illumination, J. Opt. 16 (2014), 065301-1-065301-8. 